\title{
Coil Size and Geometric Field Quality in Short Model Dipoles for LHC
}

\author{
Z. Ang, L. Bottura, S. Russenschuck, A. Sienko, D. Tomnasini, L. Walckiers \\ CERN, LHC Division, 211 Geneva 23, Swikerland
}

\begin{abstract}
We have measured the magnetic field at room temperature and at $1.8 \mathrm{~K}$ on more than twenty, $1-\mathrm{m}$ long, single aperture IHC superconducting dipole models. The magnets feature either a 5-block coil geonetry or the baseline 6-block genmetry foreseen for the I.IfC. Comparison of' warm and cold measurements show that the coil geometry is essentially unchanged during cooldown. We have therefore used mechanical mensurements taken on the coil and collars during assembly to estimate the azimuthal coil length. Based on these measurements we show hore that the sensitivity of allowed larmonics on coil size is in good agreement witl the prediction obtained from the numerical model used for designing the LIIC mugnets.
\end{abstract}

\section{INTRODUCTION}

The main bending dipoles for the Large Hadron Collicer (LIIC) must satisfy strict requirements on the magnetic field quality in order to achieve the expected benm luminosity at collision energy. This translates to tight manufacturing tolerances and requires that all magnets are systematically tested during production and at the reception at CERN [1]. The LHC dipoles are manulactured assembling four superconducting coils in a support structure formed by Laminated collars. The collared coil assembly is completed by an iron yoke and a lenk-tight shrinking cylinder of stainless steel. One of the key parameters to be controlled during production is the geometry of the coils after assembly in the collars, and in particular the azimuthal length $a$ of the coil layers. This is clefined as the length of the arc between the coil midplane and the surface of the pole (see Fig. 1). This parameter depends mainly on the pole shims between the coil and the collars and on the collar deformation during assembly, Studies on the magnets at HERA [2] and SSC [3]

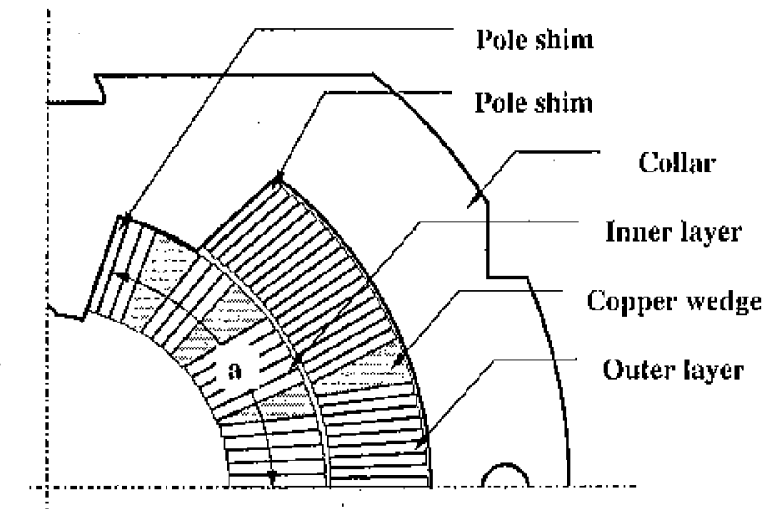

Fig. 1. Schenatic geometry of a collared coil assembly (one quadrant), and definition of the nzimuthal coil size $a$.

Matuscript teceiycd September 27, 1999 have shown that variations of the geometric harmonics are strongly correlated with coil deformations. We have reported elsewhere a first analysis of the correlation between the estimated coil size and measured allowed harmonics in $\mathrm{LHC}$ dipole models [4]. Here we extend the analysis to a larger number of magnets, featuring different coil geometries and collar materials. As done in [4] we focus on the first allowed harmonics. A good control of the allowed harmonics is very important as they will produce systematic effects in the LHC.

\section{SERIES OF MAGNETS TESTED}

The series of short models that are being manufactured at CERN within the frame of the R\&D program for the LHC main dipoles [5] provides an ideal test-bed for correlation studies. A total of $21,1-\mathrm{m}$ long, single aperture magnets (the MBSMS series) and 5 twin aperture magnets (the MBSMT series) have been produced so far. The main purpose of the short model program is to explore the influence of manufacturing paraneters on the quench performance and training behaviour. For this reason the coils of these magnets have been collared adjusting the pole shims to achieve coil compressions spanning a wide range, approximately 20 to $70 \mathrm{MPa}$ after cool-down. Consequently the azimuthal length of the coils after collaring varied from magnet to magnet. This has given a good opporiunity to study the dependence of the field harmonics on the coil size. The magnets considered here are the single aperture models MBSMS4 to MBSMS23 that we group in three families:

- magnets MBSMS4 through MBSMSI3 constitute the first family. They are built using coils with 5 blocks of cables per quadrant (sce Fig. 2) assembled into Al-alloy collars;

- the coil cross section has been modified as a result of optimization studies that have taken place during the R\&D program. Magnets MBSMS15 through MBSMS23 feature the new optimized coil, with 6-cable blocks per quadrant (see again Fig. 2). About half of these magnets, our second family, have been assembled in Al-alloy collar's (MBSMS 15 through MBSMS 18);

- the third family is formed by the remaining models, with 6-blocks coil geonetry and austenitic stecl collats. This family (MBSMS19 through MBSMS23) is at present the closest match to the baseline design of the main bending dipoles for LHC.

Several magnets were re-worked in different versions, changing the collaring and/or yoking conditions. In total we have performed magnetic measurements on approximately forty different magnets. 

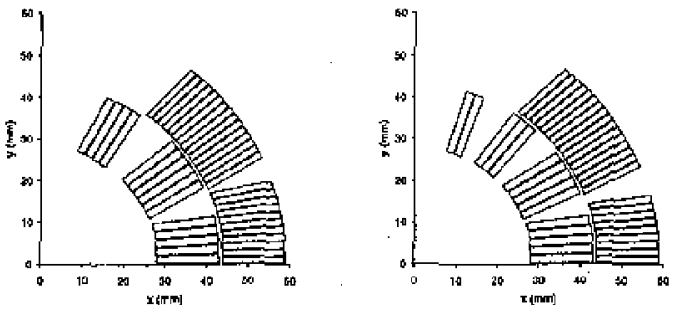

Fie. 2. Nominal geometry of one guadrant of the 5-blocks and 6-blocks dipole coils.

\section{FIELD QUALITY DEFINITIONS}

We follow standard practice in the description of the magnetic field $B$ in accelerator magnets [6]. We ignore variations along the magnet length $z$ and expand the field in the magnet cross-section $x-y$ using the complex power series:

$$
\mathbf{B}(x, y)=B_{y}+i B_{x}=10^{-4} B_{1} \sum_{y=1}^{\infty}\left(b_{n}+i a_{n}\right)\left(\frac{x+i y}{R_{r e s}}\right)^{n-1}
$$

where $B_{1}$ is the dipole strength, $R_{r e f}$ is the reference tadius (17 mm), $b_{n}$ and $a_{n}$ are the normal and skew $2 n$-pole coefficients. In accordance with the above definition the harmonic coefficients are quoted in dimensionless anits. In this paper we report the harmonics in a reference frame where the dipole is purely normal. In this reference frame the only harmonics allowed by symmetry are the normal, odd coeffieients. For the dipole component we will also quote normalized values in units, defined as follows:

$$
b_{1}=10^{4} \frac{B_{1}-B_{1}^{\text {nom }}}{B_{1}^{\text {rom }}}
$$

where $B_{t}^{\text {non }}$ is a nominal dipole field evaluated as the average of the measured walues on all magnets of a same family. With the above defiution the values of $b_{i}$ for each family are centered around the average field. We therefore neglect systematic differences anong magnet families, concentrating on relative variations within the same family,

\section{MECHANKCAL MEASUREMENTS AND COIL SIZE}

The coils used for the series of the MBSMS magnets do not have the same azimuthal size because of small variations of cable size, insulation thickness, thickness of the copper wedges and curing conditions. In order to achieve the desired azimuthal pre-compression the pole shims have been adjusted from maguet to magnet. The basis for the adjustment of the pole shims is the relation between azimuthal compression and pole displacoment established in a press. During collaring the coil is forced into the volume delimited by the collar structure. In the ideal case of infinitely rigid collars the azimuthal coil size would be simply given by $a=a_{\text {aish }}-\delta_{\text {shim, }}$ where $a_{n o m}$ is the nominal azimuthal length and $\delta_{\text {slim }}$ is the difference between the thickness of the pole shim used during collaring and the ideal shim (positive in the case of a shim larget than nominal). In reality the collars are not infintitely
TABLE I

Standard Deyiation of Tile correlation BeTweEn Warm akd Cold

\begin{tabular}{|c|c|c|c|c|c|c|}
\hline \multirow[t]{2}{*}{ Order } & \multicolumn{2}{|c|}{$\begin{array}{c}5 \text { blocks Al } \\
\sigma_{\text {tristecold }}\end{array}$} & \multicolumn{2}{|c|}{$\begin{array}{l}6 \text { blocks Al } \\
\sigma_{\text {unem tout }}\end{array}$} & \multicolumn{2}{|c|}{$\begin{array}{c}6 \text { blocks SS } \\
\sigma_{\text {warm-cuth }}\end{array}$} \\
\hline & $b_{13}$ & $a_{n}$ & $b_{4}$ & $a_{11}$ & $b_{n}$ & $a_{n}$ \\
\hline 2 & 0.25 & 2.87 & 0.16 & 0.28 & 0.49 & 1.24 \\
\hline 3 & 0.82 & 0.62 & 0.50 & 0.60 & 0.89 & 1.21 \\
\hline 4 & 0.26 & & 0.07 & 0.07 & 0.30 & 0.74 \\
\hline 5 & 0.47 & & 0.11 & 0.13 & 0.36 & 0.74 \\
\hline
\end{tabular}
GEOMETRIC FIARMONICS (IN UNTSS (9 $17 \mathrm{MM}$ )

rigid and deform elastically during collaring. The total deformation $\delta_{\text {coliar }}$ under the residual pre-compression has been measured after collaring at the outer surface of the collars, it correspondence of the poles. This measurement has been used to correct the estimated coil size as follows:

$$
a \approx a_{\text {Haia }}-\delta_{\text {srim }}+\delta_{\text {collar }} / 2 \text {. }
$$

The estimated azimuthal size difference $\delta=a-a_{\text {mom }}$ ranges from $0.1 \mathrm{~mm}$ to $\mathrm{f} \mathrm{mm}$ for the models with 5 -blocks coils, and from $-0.2 \mathrm{~mm}$ to $0.4 \mathrm{~mm}$ for those with 6-blocks coils.

\section{MAGNETIC MEASUREMENTS}

The warm and cold $(1.8 \mathrm{~K})$ measurements of the dipole model magnets are performed in a vertical test set-up, described in detail elsewhere [7]. The field is measured using radial coils mounted on a glass-fibre shaft rotating in the bore of the magnet. Five adjacent coils sections are installed to measure the field dependence along the magnet bore. Three $200 \mathrm{~mm}$ long coil sections cover the straight part. As we have done in our ear]ier study [4], we will refer here to the results from the centermost coil for the dipole component of the lield, while the higher order harmonics will be given as dipole-weighted averages over the straight part. The cold testing procedure started with a standard pre-cycle (ramp-up to $11.75 \mathrm{kA}$ and down to $50 \mathrm{~A}$ ) to achieve a known and reproducible initial state. We have then ramped the current in steps and taken measurements at constant current at approximately 20 current values on both ramp-up and rampdown powering branches. The geometric harmonics have been computed as the avernge of the measured values on the ramp-up and ramp-down branches at $5 \mathrm{kA}$ [4]. Warm measurements were performed using the same test equipment, at $30 \mathrm{~A}$ current in the magnet. Positive and togative cutrent measurements were taken to eliminate residual magnetization effects that can be significant at the small field level used during warm testing,

As reported in our previous work [4] there is a good correlation between the allowed harmonics measured in warm conditions and the geometric value in cold conditions. We strengthen our statement showing in Figs. 3 and 4 the scatter plot of warm and cold geometric sextupole and tecapole for all single aperture models tested. The correlation is excellent and demonstrates that the thermal contraction during cool-down has no influence on allowed harmonics. In Table I we report the standard deviation of the warn-cold correlations, $\sigma_{\text {worm-coid }}$ defined as in [4], for the three families of magnets. This quantity gives an estimate of the typical 


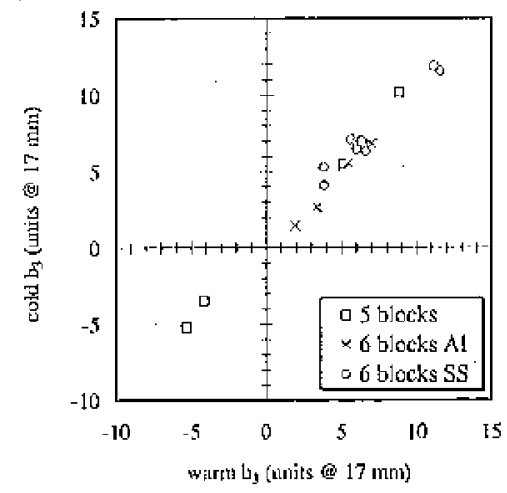

Fig. 3. Scatter plot of normal sexuppole mensured in warm and cold conditions (geometric component only).

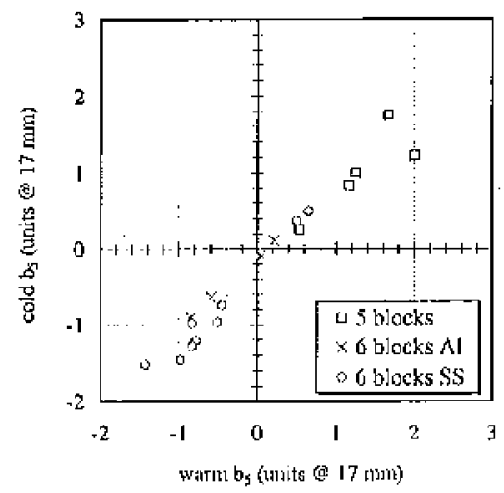

Fig, 4. Seatter plot of mormil decapole measured in warm and colel conditions (geometrie component only).

range of coil geometry control that can be achieved during series production of the dipoles, if warm measurements only are used to provide feed-back. We see there, as also evident from Figs. 3 and 4, that for allowed harmonics there is in practice no difference for different coil cross-sections or collar material. The variance observed on the non-allowed harmonics (in particular $a_{2}$ ) could be the result of a statistical anomaly due to the small number of tests (four) performed on Al-collars 6-blocks magnets.

\section{FIELD CALCULATION AND RECONSTRUCTION}

For the calculation of the effect of the azimuthal deformation of the coil we have used the analytical model of ROXIE [8]. We have assumed that the coil defornation lakes place only in the cables, and that the copper wedges between blocks behave rigidly. A change of coil size in the aximuthal direction has been simulated stretching the width of the cables by a fixed amount. The total displacement $\delta$ of the pole surface was the result of the cumulative addition of the changes of the width of the cables in a layer. The result of this calculation is the sot of harmonics $b_{n}^{\text {dfff }}+i a_{n}^{d f f}$ for the deformed coil. We have compared these values to the harmonics of the coif at nominal size $b_{n}^{\text {wirt }} \bullet i a_{n}^{\text {nona }}$ and we have computed the gradient (Jacobian) of the allowed harmonics with respect to the displacement:
TABLE []

HARMONICS J ACOBIANS FOR AN AZIMUTHAL DISPLACEMENT OF TILE POLE SURFACE (IN UNJTS @ 17 MM/MM DISPLACEMLNT)

\begin{tabular}{ccccc}
\hline Order & \multicolumn{2}{c}{5 blocks coil } & \multicolumn{2}{c}{ 6 blocks coil } \\
& Inuer layer & Outer layer & Inner layer & Outer bayer \\
\hline $\mathbf{b}_{1}$ & -54 & $-4 \mathbf{1}$ & -55 & -42 \\
$\mathbf{b}_{5}$ & -16.1 & -13.5 & -17.2 & -12.5 \\
$\mathbf{b}_{5}$ & 3.6 & 0.52 & 3.6 & 0.7 \\
$\mathbf{b}_{7}$ & -1.7 & 0.16 & -1.3 & 0.14 \\
$b_{9}$ & 0.97 & -0.02 & 0.48 & -0.02 \\
\hline
\end{tabular}

$$
\frac{\partial b_{n}}{\partial \delta} \approx \frac{b_{n}^{\text {def }}-b_{n}^{\text {new }}}{\delta} .
$$

This gradient quantifies the sensitivity of the harmonic $b_{n}$ to azimuthal coil size variations. Separate calculations were performed for the inner and outer layer, and for different amplitudes of displacement of the pole to confirm that fo: small displacements the relation between pole displacement and allowed harmonics is linear. The results of these simulations are summarized in Table II, where we report the Jacobians of the transfer function and of allowed harmonics with respect to a symmetric, outward displacement of all the pole surfaces in azimuthal direction: As expected, low order harmonics are the most affected. In addition the low order harmonics of both coil geometries depend strongly on both inner and outer layer size, while higher order harmonics are only sensitive to variations of the inner layer size. Comparing the values in Table II we remark finally that, apart for $b_{9}$, the Jacobians for the two coil types are essentially the same.

We have computed the geometric barmonics of all magnets tested using the estimated coil size of inner and outer layer, $\delta_{\text {inner }}$ and $\delta_{\text {outer }}$ respectively, and the Jacobians from Table I:

$$
b_{n}=b_{n}^{\text {ustrit }}+\left.\delta_{\text {irtrter }} \frac{\partial b_{n}}{\partial \delta}\right|_{\text {innler }}+\left.\delta_{\text {ottert }} \frac{\partial b_{n}}{\partial \delta}\right|_{\text {putter }} .
$$

The computed geometric dipole, sextupole and decapole are compared in Figs. 5, 6 and 7 to the geometric values measured in cold conditions. The reconstruction agrees satis factorily with the measurements. To quantify the quality of the correlation we have fitted each family of magnets with an ideal correlation line (unit slope) and adjusted offset $\Delta_{\text {catle }}$ The values of the offsets for the three families of magnets are reported in Table III. Note that in accordance with the definition of the dipole variation given by (2) we have neglecled systematic offsets on $b_{r}$. For this reason dipole offsets are not reported in Table III.

For an idcal correlation we expect $\Delta_{\text {calc }}=0$. A value different from zero points to systematic effects that have not been taken into account in the reconstruction. We see in Table III and in Fig. 6 that for the sextupole there is a clear trend of decreasing offset $A_{\text {calc }}$ in going from 5 blocks coils to 6 blocks coils, and further from Al-alloy to austenitic steel collars. We believe that the offset is largely due to an additional systematic deformation of the collars during assembly. This deformation results in a deviation of the final coil geometry from the nominal one. The effect is stronger if Al is used as collar material because its elastic modulus is 
smaller than that of austenitic steel. A similar behaviour is observed for $b_{5}, b_{7}$ and $b_{9}$ (see Table III). In this respect steel collars are superior as the final coil geometry is closer to the nominal one.

A second quantity of interest is the spread around the ideal correlation line. We quantify this spread using the standard deviation around the fit line $\sigma_{\text {cotr }}$, defined as in [4] and reported in Table III for the three families. This quantity gives an overall measure of random variations from magnet to - magnet that can be associated with uncertainties in the reconstruction, changes of manufacturing parameters (e.g. coil pre-stress) or components (e.g. different radial shims). It is therefore representative of the typical control that can be achieved throughout a production once systematic effects are corrected. T'he three families do not show particular differences in this respect. Note that in our case large geometry vatiations were planned from the start of the production of the model dipoles, therefore the values of $\sigma_{\text {colls }}$ quoted in Table III should be regarded as a conservative upper bound of the standard deviation for the series production of the LHC dipoles.

\section{CONCLUSIONS}

In the LHC magnets the azimuthal coil size after collaring, in warm conditions, correlates well with allowed geometric harmonics measured in cold conditions at $5 \mathrm{kA}$ excitation current. This is a direct consequence of the fact that irrespective of the coil geometry or collar material the coil geometry is not deformed during cool-down, except for a uniform thermal shrinkage. The sensitivity of the geometric harmonics to azimuthal size variations can be predicted accurately using an analytical model. Examining the three families of models tested it can be seen that a clear advantage of austenitic steel collars is that the coil geometry of the finished magnet is closer to the nominal one owing to the larger structural rigidity of steel.

\section{REFERENCES}

[1] L. Walckiors et ail, "Towarts series mensuretneth of the LHC superconducting dipole mangets", Proc. of 1997 Path Acc. Conf., Vancouver, pp. 3377-3379, 1997.

[2] K.-H. Mess, P, Schmmeser, S, Wolf, Superconducling Accelerator Magnets, World Scientifi, 1996.

[3] T. Ogitsu, A. Devred, "Influence of azimnilat coil size variations on magnetic ficld lacmonics of superconducting particle accelerater magnets", Rev. Sci. Inst, val. 65 (6), PP. 1998-2005, 1994 .

[4] Z. Ang, L. Botturn, D. Tommnsini, L. Walckiers, "Magnetic ficld quality of short superconducting dipole model magnets for LHC', Ptow, of $15^{\text {th }}$ Int. Mag, Tech, Conf, pp. 145-148, 1997.

[5] N. Andreew et al., "The 1-m long simple apotture dipole coil test progtam for LHC", Proc. of 1996 European Part. Asc Conf., Sitges, pp. 2258-2260, 1996.

[6] R.A. Beth, "Complex representation and computation of twodimensional magnetic fields", J. Appl. Phys, wol. 37 (7), Pp. 2568-2571, 1966.

[7] Z. Ang, I. Lejar, L. Bottura, D. Richter, M. Sheehan, L. Walckicrs, R. Wolf, "Measurement of AC loss and magtotic ficld duting tamps in the LHC modal dipotes", IEEE Trans. Appl. Sup, vol. 9 (2), pp.742-745, 1999.

[8] ROXIE: Rouline for the optitnization of magnet X-sections, inverse field calculation and coll and design, $S$. Russenschuck ed, CERN Yellow Report 99-01, 1999.
TABLE III

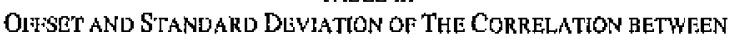
GROMITTRIC HARMONICS AND RECONSTRUCTION (IN UNITS AT 17 MM)

\begin{tabular}{lcccccc}
\hline Orcter & \multicolumn{2}{c}{5 blocks Al } & \multicolumn{2}{c}{$\sigma$ blocks Al } & \multicolumn{2}{c}{ 6 blocks SS } \\
& $\Delta_{\text {cinc }}$ & $\sigma_{\text {cnlo }}$ & $\Delta_{\text {cnlc }}$ & $\sigma_{\text {chlc }}$ & $\Delta_{\text {cils }}$ & $\sigma_{\text {calc }}$ \\
\hline 1 & & 7.0 & & 22.9 & & 15.5 \\
3 & $14.5 \pm 0.9$ & 2.9 & $10.5 \pm 0.5$ & 1.6 & $4.5 \pm 0.9$ & 2.5 \\
5 & $0.4 \pm 0.3$ & 1.0 & $-1.0 \pm 0.1$ & 0.4 & $0 \pm 0.1$ & 0.4 \\
7 & $0.7 \pm 0.07$ & 0.2 & $0.5 \pm 0.1$ & 0.3 & $0.2 \pm 0.05$ & 0.2 \\
9 & $-0.2 \pm 0.06$ & 0.2 & $-0.1 \pm 0.02$ & 0.1 & $-0.06 \pm 0.01$ & 0.04 \\
\hline
\end{tabular}

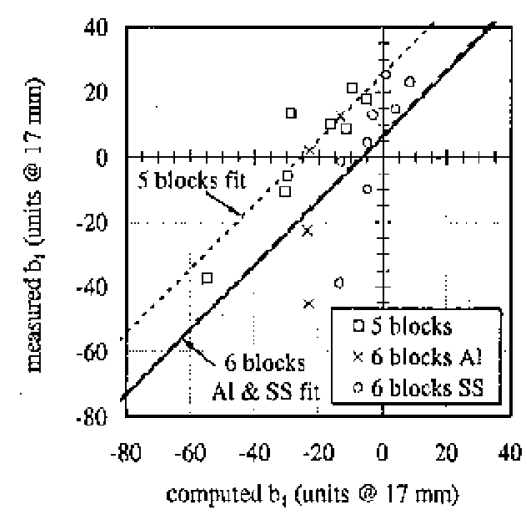

Fig. 5. Scatter plot of the variation of nomal dipole mensured and reconstructed by calculation in all models tested.

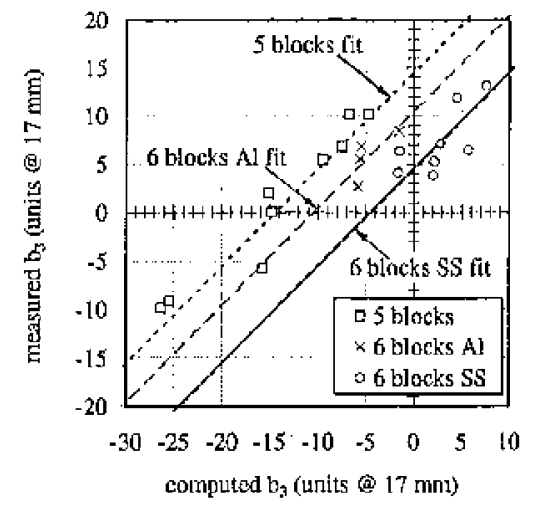

Fig. 6. Scatter plot of measuted and reconstructed $b_{3}$

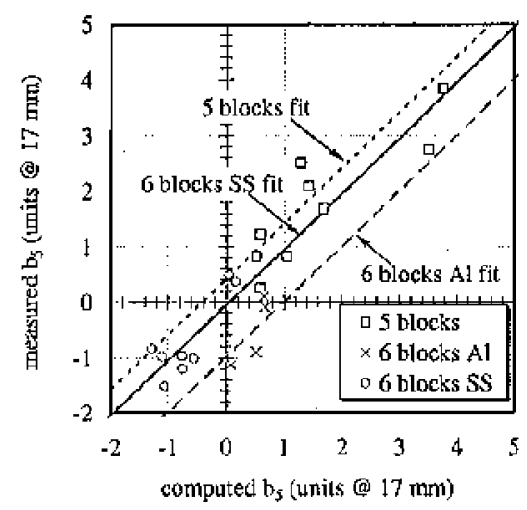

Fig. 7. Scatter plot of measured and reconstructed $b_{5}$. 